\title{
Identification of the D-S evidence conflict based on multi-source information
}

\author{
NAN Feng \\ Hebei Polytechnic University \\ Tang shan, China \\ e-mail: bluenanfengly@163.com
}

\author{
LI Yang \\ Hebei Polytechnic University \\ Tang shan , China \\ e-mail: liyang@heuu.edu.cn
}

\begin{abstract}
TO solve the applying constraints problem of D-S evidence conflict in multi-information fusion and achieve the systematic identification of evidence conflict, we introduce the $\mathrm{K}-\mathrm{L}$ information on the distance function to describe the characteristics of the conflict between the evidence in the paper, construct the distance matrix defined the level of conflict of independent evidence in the whole system. Simulating results show that: the effective conflict identification of K-L information distance can complete the application constraint of D-S theory's synthetic rule, obtaining the optimized convergence results for synthesis of normal conflicting evidences, isolating the highly conflicting evidence.
\end{abstract}

Keywords-Dempster-Shafter fusion; information distance; conflict characteristics; identification

\section{INTRODUCTION}

Dempster-Shafer (DS) theory of evidence from the 1960s multifunctions has reasoning ability with uncertain information and has good practical performance on data fusion and information prediction. D-S evidence combination rule is the core principle of Dempster-Shafer (DS) theory of evidence. If the information is reliable, we can use the theory updating the truth of evidence. We can use the mutual support and complement between evidence improving the accuracy of information synthesis result. However, in reality, there are varying degrees of conflict (Inconsistent information) between the evidence due to interference of the inclement objective environment and subjective emotional factors, which causing the defects of D$S$ evidence theory. So, the identification of evidence conflict characteristic and the information fusion method in conflict become the core research of Dempster-Shafer (DS) theory of evidence.

We can realize the optimal fusion of DS theory under the conflicting condition of system evidence because of the algorithm about conflict coefficient $\mathrm{K}$ in D-S evidence combination rule Introduced and the normalization factor of $\mathrm{K}$ used for amending the synthesis of information. Because there is no feature analysis and information extraction about the evidence conflict in the entire system, it caused incomplete information in the integration process of evidence system. In this paper, we introduced the distance function of the K-L to replace the D-S conflict coefficient $\mathrm{K}$, so as to illustrate the level of evidence conflict in the system. We define the degree of K-L evidence conflict in order to identify the conflict feature and optimize the scope and convergence performance of DS evidence fusion theory. we can improve the reliability and sensitivity of system prediction by means of analyzing the conflicting information.

\section{D-S EVIDENCE THEORY AND EVIDENCE CONFLICT}

\section{A. Preliminaries of the D-S evidence theory}

The D-S theory assumes a non-empty set $\Theta$, to describe all elements with mutually exclusive and exhaustive in the constitute space, $\Theta=\left\{A_{1}, A_{2} \cdots A_{N}\right\}$ called the frame of discernment. Element is a collection of $\mathrm{N}$ mutually exclusive $2^{N}$ subset, denoted by $2^{\Theta}$, quality of evidence (Mass) is the trust to support the proposition in DS theory.

Definition $1^{[1]}$ Set $\Theta$ is the identification of D-S theory framework, comprehensive and mutually exclusive propositions composed of power set function $m: 2^{\Theta} \rightarrow[0,1]$, and suffice

$$
m(\Phi)=0, \quad \sum_{A \subset \Theta} m(A)=1
$$

$m(A)$ is the basic probability assignment function under the framework $\Theta$ of $A$, On behalf of the trust and support of evidence, While $m(A) \neq 0$, A is the focal element of BPA ( focus on evidence $\mathrm{A}$ ).

Definition $2^{[1]}$ Support or belief function (bel) on behalf of the total trust to proposition A with the decision-makers,

$$
\operatorname{Bel}(A)=\sum_{B \subseteq A} m(B), \quad A \subseteq \Theta, B \neq \Phi
$$

Belief function is applied to a collection and all subsets, Bel-based on the global trust.

D-S theoretical composition rules:

Evidences $M_{1} 、 M_{2}$ in one framework $\Theta$,

$\mathrm{BPA} \rightarrow \mathrm{m}_{1} 、 \mathrm{~m}_{2}$, the focal elements are $\left(A_{1}, \cdots, A_{k}\right)\left(B_{1}, \cdots, B_{l}\right)$, assumes:

$$
K=\sum_{A_{i 1} \cap B_{j 2}=\Phi} m_{1}\left(A_{i}\right) m_{2}\left(B_{j}\right)
$$

$\mathrm{K}$ is the conflict coefficient, characterization the degree of contradictions between Evidences in combination of D-S.

Evidence Fusion results: 


$$
\begin{aligned}
m(C) & =\mathrm{m}_{1} \oplus m_{2}=m_{1}(A)+m_{2}(B) \\
& =\frac{1}{1-K} \sum_{A_{1} \cap B_{j 2}=C} m_{1}\left(A_{i}\right) m_{2}\left(B_{j}\right) \quad \forall \mathrm{C} \subset \Theta, \mathrm{C} \neq \Phi
\end{aligned}
$$

$m(C)$ is the new evidence assignment after the orthogonal combination; while $A_{i 1} \subseteq B_{j 2}$ then $C_{i}=A_{i 1}$, Indicates information consistent part of an element between the evidences; while $A_{i 1} \cap B_{j 2}=\Phi$, elements inconsistent information and expressed as a conflict. $\oplus$ is Orthogonally,

(1-K) as the normalization factor which excluding conflict interference.

When exists the $\mathrm{n}$ evidences,

$$
\begin{gathered}
m(A)=\mathrm{m}_{1} \oplus m_{2} \oplus \cdots \oplus m_{n} \\
=\frac{1}{1-K} \sum_{A_{i 1} \cap A_{i 2} \cap \cdots \cap A_{i n}=A}\left(A_{i}\right) m_{2}\left(A_{i}\right) \cdots m_{n}\left(\mathrm{~A}_{\mathrm{i}}\right) \quad \forall \mathrm{A} \\
K=\sum_{A_{i 1} \cap A_{i 2} \cap \cdots \cap A_{i n}=\Phi}\left(A_{i}\right) m_{2}\left(A_{i}\right) \cdots m_{n}\left(A_{i}\right)
\end{gathered}
$$

\section{B. The evidence conflicts's proposed}

1984, Zadch ${ }^{[1]}$ examples demonstrate the drawbacks of the normalization factor, noting the application restrictions under conflict of evidence in Dempster combination rule ${ }^{[2]}$. On the basis of the Zadch ,constructed three complex paradox situation by application on the Dempster combination rule, and obtained instance demonstration on Studies of substantial evidence conflict ${ }^{[3,4]}$.

To the conflict coefficient $\mathrm{K}$ :

- $\mathrm{K}=1$, evidences $\mathrm{m} 1 、 \mathrm{~m} 2$ are completed conflicts, D$\mathrm{S}$ theoretical composition rules fails;

- $\mathrm{K}=0$, The evidence information completely consistent, application the Dempster synthesis can achieve fast focus;

- $\mathrm{K} \rightarrow 1$, There is a high degree of conflict between the evidences, the fusion result may appear a paradox;

- $\quad 0<\mathrm{K}<1$, Two evidences partially compatible.

Hence, any evidence $(K \neq 0)$ combination will be results varying degrees of impact due to the existence of the conflict of fusion.

To solve the above problems of D-S fusion theory in the practical application of evidence feature conflict which can lead to systems integration errors or reduced efficiency, this paper introduces the K-L information distance express distribution difference between the evidences, trying to provides a viable solution for the evidence theory under the features conflict from independent evidence to the evidence system Perspective

\section{EXPRESSION OF THE K-L INFORMATION DISTANCE IN EVIDENCE CONFLICT}

Ideally, the assignment trust of the evidence in the same frame of discernment can get the support from the information by the ambient monitoring, and determined (increase) the fusion results by the ability of focus fusion. In practical applications, due to the actual existence of the conflict and interference, should be identified for all the conflict among the evidences and reasonable allocation. Conflicts coefficient $\mathrm{K}$ of D-S evidence theory to be able to describe the paradoxical situation of information between the two evidences, but to more evidences fusion and the new assignment functions were orthogonal increased fusion difficult. Combining orthogonal can characterization evidence conflict level of the overall system, but can not be specific expression conflict of a single evidence to the system, bring application limitation to the conflict feature recognition of evidence fusion theory. The concept of K-L information distance express the degree of conflict between the evidences, through matrix combination to identify conflicts of evidence characteristics in the entire fusion system.

\section{A. The concept of K-L information distance}

K-L information distance proposed by the statistician Kullback and Leibler in the middle of the 20th century, used to determine the closeness of the two probability distributions.

Definition $3^{[5]}$ In one framework $\Theta$, the BPA of evidences $\mathrm{M}_{1} 、 \mathrm{M}_{2} \rightarrow m_{1}(x) m_{2}(x)$, information distance from $M_{1}$ to $M_{2}$ is:

$$
I\left(M_{1}: M_{2}\right)=I\left(m_{1}: m_{2}\right)=\int_{\Theta} m_{1}(x)\left|\ln \left[\frac{\mathrm{m}_{1}(x)}{\mathrm{m}_{2}(x)}\right]\right| d x
$$

According to D-S evidence theory, $m_{1}(x)=m_{1}\left(A_{i}\right), m_{2}(x)=m_{2}\left(B_{j}\right)$. In the comprehensive framework $\Theta$, focal element number of the evidence M1 M2 is same, as $i=j=1, \cdots, k$. Due to the discrete form of BPAfunction, the formula (5) is expressed :

$$
\underset{B P A}{I}\left(m_{1}: m_{2}\right)=\sum_{i=j=1}^{k} m_{1}\left(A_{i}\right)\left|\ln \left(\frac{m_{1}\left(A_{i}\right)}{m_{2}\left(B_{j}\right)}\right)\right|
$$

Definition 3 shows, $I\left(m_{1}: m_{2}\right) \geq 0$, and only $A_{i}=B_{j}$ then $I\left(m_{1}: m_{2}\right)=0$. Greater the K-L information distance, greater the difference of probability assignment function between the two pieces of evidence, but the smaller in the contrary. When the two evidence fully consistent, $\mathrm{I}=0$. Therefore, K-L information distance and D-S conflict coefficient $\mathrm{K}$ has the same meaning, by describing the differences in the distribution of BPA function to reflect the degree of conflict among the evidences. 
B. Identifying of evidence conflict by the K-L information distance

Evidences of plurality of uniformly distributed (random) in limited temporal,

$M_{i}, i=1,2, \cdots, n$. Command $I_{i, j}=I\left(m_{i}: m_{j}\right)$, generally

$I_{i j}\left(m_{i}: m_{j}\right) \neq I_{j i}\left(m_{j}: m_{i}\right)$, then $I_{i j}$ express the information

distance from evidence $\mathrm{j}$ to $\mathrm{i}$, structure matrix of $\mathrm{K}-\mathrm{L}$

information distance:

$$
\left(\begin{array}{ccccc}
I_{11} & I_{12} & \cdots & \cdots & I_{1 n} \\
I_{21} & I_{22} & \cdots & \cdots & I_{2 n} \\
\vdots & \vdots & \vdots & \vdots & \vdots \\
I_{n 1} & I_{n 2} & \cdots & \cdots & I_{n n}
\end{array}\right)
$$

In the selected area, total information distance of all evidences to the evidence is:

$$
I_{i}=\sum_{j=1}^{n} I_{i, j}
$$

Obviously, when $i=j, I_{i j}=0 . \quad I_{i}$ characterized the degree of difference of the distribution function from evidence $i$ to all the evidence in the integration system. In $n$ evidences existing monitoring system, the reliable evidence information not only need to get adjacent evidence support, in the system steady-state and other evidence also should keep reasonable difference, so the total information distance performance is smaller; Some with adjacent evidence consistent with the evidences, evidence of differences in the total information distance corresponding amplification, may be due to local change; The largest total information distance are those evidences with other evidences that highlight the differences, may be situation mutation or test failure cause. Therefore, based on the comprehensive analysis on some evidence to the all the evidence of the total information distance, can describe the status of the conflicts in the whole system of evidence $i$.

Definition 4 In order to manifest the influence degree of conflict among the evidences in the whole system, through the distribution difference in the proportion of the overall difference, defined distribution difference rate $H_{i}$ is:

$$
H_{i}=\frac{I_{i}}{\sum_{i=1}^{n} I_{i}} \quad i=1,2, \cdots, n .
$$

Formula (8) called $H_{i}$ as evidence conflict degree based on $\mathrm{K}-\mathrm{L}$ distance information (hereinafter referred to $\mathrm{K}-\mathrm{L}$ evidence conflict degree). When evidence I and other evidences of function distribution difference is bigger, the distance information in the system of the total gap "contribution", describe the conflict characteristics more obvious. Therefore, K- L evidence conflict degree can representation independent evidence $i$ and other evidence of the conflict features, also can show the total conflict the influence of position of evidences in the whole system.

\section{SIMULATION EXPERIMENT}

A objective monitoring system In the limited time and space, assumed the frame of discernment $\Theta=\{A, B, C\}$. There collected eight evidences, its BPA function are as follows $m(A) 、 m(B)$ 和 $m(C)$, and satisfying $\sum_{\bullet \subset \Theta} m_{i}(\bullet)=1$

$$
\begin{array}{lll}
m_{1}(A)=0.5, & m_{1}(B)=0.2, & m_{1}(C)=0.3 ; \\
m_{2}(A)=0.01, & m_{2}(B)=0.89, & m_{2}(C)=0.1 ; \\
m_{3}(A)=0.55, & m_{3}(B)=0.1, & m_{3}(C)=0.35 ; \\
m_{4}(A)=0.55, & m_{4}(B)=0.1, & m_{4}(C)=0.35 ; \\
m_{5}(A)=0.45, & m_{5}(B)=0.15, & m_{5}(C)=0.4 ; \\
m_{6}(A)=0.1, & m_{6}(B)=0.8, & m_{6}(C)=0.1 ; \\
m_{7}(A)=0.6, & m_{7}(B)=0.15, & m_{7}(C)=0.25 ; \\
m_{8}(A)=0.5, & m_{8}(B)=0.25, & m_{8}(C)=0.25
\end{array}
$$

$\mathrm{K}-\mathrm{L}$ information distance matrix, and calculated by the formula (6) - (8):

TABLE $1 \mathrm{~K}-\mathrm{L}$ INFORMATION DISTANCE AND EVIDENCE CONFLICTS DEGREES BASED ON EVIDENCE FUSION

\begin{tabular}{c|cccccccc|c|c}
\hline & I1 & I2 & I & I4 & I5 & I6 & I7 & I8 & Ii & Hi \\
\hline I1 & 0 & 1.987 & 0.045 & 0.045 & 0.024 & 0.857 & 0.021 & 0.010 & 2.988 & 0.084 \\
I2 & 1.179 & 0 & 1.780 & 1.780 & 1.408 & 0.072 & 1.452 & 0.999 & 8.672 & 0.243 \\
I3 & 0.037 & 2424 & 0 & 0 & 0.023 & 1.168 & 0.029 & 0.078 & 3.760 & 0.105 \\
I4 & 0.037 & 2424 & 0 & 0 & 0.023 & 1.168 & 0.088 & 0.078 & 3.819 & 0.107 \\
I5 & 0.025 & 2.000 & 0.024 & 0.02 & 0 & 0.980 & 0.058 & 0.064 & 3.172 & 0.089 \\
I6 & 0.838 & 0.145 & 1.368 & 1.37 & 1.050 & 0 & 1.068 & 0.678 & 6.517 & 0.183 \\
I7 & 0.002 & 2510 & 0.005 & 0.11 & 0.008 & 1.145 & 0 & 0.033 & 3.813 & 0.107 \\
I8 & 0.010 & 1.868 & 0.097 & 0.1 & 0.063 & 0.743 & 0.036 & 0 & 2.918 & 0.082 \\
\hline
\end{tabular}

Table 1 shows the evidence K-L information distance from evidence $\mathrm{j}$ to the $\mathrm{i}, I_{i}$ indicates the distance sum of all the evidence to the $\mathrm{i}$-th evidence, based on the conflict in the $\mathrm{K}$-L information distance the evidence $\mathrm{i}, H_{i}$ is calculated by the proportion of the total system gap feature level.

D-S synthesis under the rules of multi-objective evidence fusion complex system can only calculate the total conflict coefficient $\mathrm{K}$, in outstanding condition of the whole system, can not single evidence like K-L reliability fusion calculated conflict feature recognition by independent evidence. The formula (4)、 (6-8) ,calculated D-S conflict coefficient K and $\mathrm{K}-\mathrm{L}$ information conflicts degrees, table 2:

TABLE 2 D-S CONFLICT COEFFICIENT K AND K-L EVIDENCE DEGREES

\begin{tabular}{cc}
\hline $\mathrm{m} 1, \mathrm{~m} 2, \cdots, \mathrm{m} 8$ & conflicts coefficient \\
\hline $\mathrm{D}-\mathrm{S}$ & $\mathrm{K}=0.65687$ \\
& $H 1=0.08381 ; \mathrm{H} 2=0.24318 ; \boldsymbol{H} 3=$ \\
$\mathrm{K}-\mathrm{L}$ & $0.10544 ; \mathrm{H} 4=0.10710 ;$ \\
& $H 5=0.08894 ; \mathrm{H} 6=0.18277 ; \mathrm{H} 7=$ \\
& $0.10693 ; \mathrm{H} 8=0.08182$.
\end{tabular}


Derived the evidence conflicts degrees from Table $2 \mathrm{~m} 2$, $\mathrm{m} 6$ and other evidence of the system existed quite different.

\begin{tabular}{|c|c|c|}
\hline D-S & conflicts coefficient & Bel of fusion results \\
\hline m1, m2, $\cdots$, m8 & $K=0.65687$ & $\begin{array}{c}m(a)=0.542817 \\
m(b)=0.21294 \\
m(c)=0.244243 \\
m(\Theta)=0\end{array}$ \\
\hline $\begin{array}{l}\text { m1,m3,m4,m5,m7,m8 } \\
\text { （isolated m2 ,m6） }\end{array}$ & $K=0.52087$ & $\begin{array}{c}m(a)=0.95644 \\
m(b)=0.00053 \\
m(c)=0.04303 \\
m(\Theta)=0\end{array}$ \\
\hline
\end{tabular}

From Table 3, the evidence existed the high degree of conflict characterized, the system direct integration can not be satisfied with the convergence effect, the fusion results is difficult to make a valid judgment; But obtained satisfaction focus fusion after the isolation of the singular points of conflict, can provide policymakers with valuable information to the judge.

\section{CONCLUSION}

The evidence conflict is the important constraint condition of D-S theory of evidence in information fusion. If we only eliminate evidence conflict, it will make information incomplete. If we don't consider the evidence conflict, it will not get structural convergence. By means of constructing probability function of evidence, we can explain the evidence conflict degree in evidence system which can't be expressed by the orthogonal conflict coefficient of K. So we can identify the conflict feature of evidence for each. we can extend the scope of the application of the theory on the basis of the identification of system conflicts and get the satisfactory decision result; we can improve the reliability of the forecasting system and avoid the loss of useful information from isolating the highly conflicting evidence.

\section{REFERENCES}

[1] Zadeh L A . Review of books : A mathematical theory of evidence[J]. AI Magazine, 1984, 5(3):81-83.

[2] Zhang S D, Wang L D. Absurdities about Dempter-Shafer's rule of combination of evidence $[\mathrm{J}]$. Systems Engineering-Theory \& Practice, 1997, 17(5):82-85.

[3] Li W Y, Guo K H. Combination rules of D-S evidence theory and conflict problem $[\mathrm{J}]$. Systems Engineering-Theory \& Practice , 2010, 30(8): 1422-1432.

[4] Liang C Y, Chen Z M, Huang Y Q, et a1. A method of dispelling the absurdities of Dempster-Shafer's rule of combination[J]. Systems Engineering-Theory \& Practice, 2005, 25(3) : 7-12.

[5] REN Kai-jun, WU Meng-da, LIU Qi. The combination of prior distributions based on Kullback information[J].Journal of the Academy of Equipment Command \& Technology, 2002, 13(4): 9092. 